\title{
Correction to: Theory of Atom Scattering from Surface Phonons: Basic Concepts and Temperature Effects
}

\author{
Correction to: \\ Chapter 7 in: G. Benedek and J. P. Toennies, \\ Atomic Scale Dynamics at Surfaces, \\ Springer Series in Surface Sciences 63, \\ https://doi.org/10.1007/978-3-662-56443-1_7
}

Equations (7.33a) and (7.82) of Chapter 7 were incorrect. These had been corrected. 